OPEN ACCESS

Edited by:

Sadri Znaidi,

Réseau International des Instituts

Pasteur, France

Reviewed by:

Teresa OMeara,

University of Michigan, United States

Sascha Brunke,

Leibniz Institute for Natural Product

Research and Infection Biology,

Germany

${ }^{*}$ Correspondence:

Adnane Sellam

adnane.sellam@umontreal.ca

Specialty section:

This article was submitted to

Fungal Pathogenesis,

a section of the journal

Frontiers in Cellular and

Infection Microbiology

Received: 03 September 2021 Accepted: 26 October 2021

Published: 19 January 2022

Citation:

Henry M, Burgain A,

Tebbji F and Sellam A (2022)

Transcriptional Control of

Hypoxic Hyphal Growth

in the Fungal Pathogen

Candida albicans.

Front. Cell. Infect. Microbiol. 11:770478.

doi: 10.3389/fcimb.2021.770478

\section{Transcriptional Control of Hypoxic Hyphal Growth in the Fungal Pathogen Candida albicans}

\author{
Manon Henry ${ }^{1}$, Anaïs Burgain ${ }^{2}$, Faiza Tebbji ${ }^{1}$ and Adnane Sellam ${ }^{1,3 *}$ \\ ${ }^{1}$ Montreal Heart Institute, Université de Montréal, Montréal, QC, Canada, ${ }^{2}$ Department of Microbiology, Infectious Diseases \\ and Immunology, Faculty of Medicine, Université Laval, Quebec City, QC, Canada, ${ }^{3}$ Department of Microbiology, Infectious \\ Diseases and Immunology, Faculty of Medicine, Université de Montréal, Montréal, QC, Canada
}

The ability of Candida albicans, an important human fungal pathogen, to develop filamentous forms is a crucial determinant for host invasion and virulence. While hypoxia is one of the predominant host cues that promote $C$. albicans filamentous growth, the regulatory circuits that link oxygen availability to filamentation remain poorly characterized. We have undertaken a genetic screen and identified the two transcription factors Ahr1 and Tye7 as central regulators of the hypoxic filamentation. Both ahr 1 and tye 7 mutants exhibited a hyperfilamentous phenotype specifically under an oxygen-depleted environment suggesting that these transcription factors act as negative regulators of hypoxic filamentation. By combining microarray and ChIP-chip analyses, we have characterized the set of genes that are directly modulated by Ahr1 and Tye7. We found that both Ahr1 and Tye7 modulate a distinct set of genes and biological processes. Our genetic epistasis analysis supports our genomic finding and suggests that Ahr1 and Tye7 act independently to modulate hyphal growth in response to hypoxia. Furthermore, our genetic interaction experiments uncovered that Ahr1 and Tye7 repress the hypoxic filamentation via the Efg1 and Ras1/Cyr1 pathways, respectively. This study yielded a new and an unprecedented insight into the oxygen-sensitive regulatory circuit that control morphogenesis in a fungal pathogen.

Keywords: Candida albicans, hypoxia, filamentation, transcriptomics, ChIP-chip, transcription factor

\section{INTRODUCTION}

Candida albicans is an opportunistic fungus and one of the most common causes of systemic fungal infection in humans with high mortality rates of $50 \%$ or greater despite currently available antifungal therapy (Sellam and Whiteway, 2016; Fisher et al., 2020). The ability of this yeast to cause infections is centrally related to different intrinsic virulence attributes such as biofilm formation, adherence to the host epithelia, the yeast-to-hyphae switch, secretion of hydrolytic enzymes (e.g. lipases, phospholipases and proteinases) and the cytolytic peptide Candidalysin (Brunke et al., 2016; Naglik et al., 2019). Of particular importance, a transition from the yeast morphology to the filamentous form is a key virulence determinant dedicated to the invasion of host tissues and, allowing to escape from phagocytes (Austermeier et al., 2020). Hyphal cells are also characterized by an enhanced adhesiveness to mucosal surfaces and are essential for the 
compression strength of C. albicans biofilms (Jacobsen et al., 2012; Wall et al., 2019). Additionally, as in Saccharomyces cerevisiae, filamentation might allow $C$. albicans to conquer niches where nutrient conditions are not limiting (Shapiro et al., 2012; Arkowitz and Bassilana, 2019).

C. albicans yeast-to-hyphae transition is controlled by an intertwined regulatory circuit that signals different filamentation stimuli encountered in distinct host microenvironments including $\mathrm{pH}$, serum, $\mathrm{N}$-acetylglucosamine, temperature, nutritional stress, hypoxia and $\mathrm{CO}_{2}$ (Villa et al., 2020). Hypoxia, the dominant conditions that C. albicans encounters inside the human host, promotes filamentation, however, the contributing mechanisms remain poorly characterized. In response to both $\mathrm{CO}_{2}$ and hypoxia, Ofd1, a prolyl hydroxylase, contribute to the maintenance of hyphae elongation by stabilizing the transcriptional activator of hypha-specific genes, Ume6 (Lu et al., 2013). The transcription factors Efg1 and Bcr1 act both as repressors of filamentation under hypoxic environments to sustain the commensal growth of $C$. albicans at temperatures $\leq 35^{\circ} \mathrm{C}$ that are slightly below the core body temperature (i.e. $<37^{\circ} \mathrm{C}$ ) (Setiadi et al., 2006; Desai et al., 2015). In opposite to Efg1-Bcr1 regulatory axis, the transcription factor Ace2 promotes filamentation under hypoxia (Mulhern et al., 2006) but also in response to the hyphae-promoting growth medium, Spider (Noble et al., 2010). Many other regulatory proteins were also essential for the C. albicans morphogenesis under hypoxia, however, they were also required to signal in response to other filamentation cues (Jr et al., 1999; Sellam et al., 2010; Spiering et al., 2010; Stichternoth et al., 2011; Bi et al., 2018; Burgain et al., 2019; Rastogi et al., 2020).

So far, there are no known regulatory circuits that mediate filamentation exclusively in response to hypoxia. This could be explained by the fact that the $C$. albicans morphogenesis regulatory pathways might be evolutionary optimized to integrate different combinations of filamentation cues to effectively promote invasive growth and virulence. In this study, we performed a quantitative analysis of gene deletion mutants from different collections of protein kinases and transcriptional regulators in C. albicans to identify specific regulators of the hypoxic filamentation (Homann et al., 2009; Blankenship et al., 2010; Noble et al., 2010; Vandeputte et al., 2012). Our results supported the aforementioned hypothesis as the majority of mutants with filamentation defects under hypoxia were previously shown to exhibit the same phenotype in response to other hyphae-promoting cues. Our work uncovered two transcription factors, Ahr1 and Tye7, that act as prominent regulators of $C$. albicans filamentation specifically under hypoxia. In summary, we used genome-wide transcriptional profiling and promoter occupancy to characterize both Ahr1 and Tye7 regulons associated with the hypoxic filamentation in C. albicans. Our data show that both Ahr1 and Tye7 act as negative regulators of the hypoxia-induced filamentation by modulating a different set of genes. Our genetic epistasis analysis supports our genomic finding and suggests that Ahr1 and Tye7 act independently to modulate hyphal growth in response to hypoxia.

\section{RESULTS AND DISCUSSION}

\section{Screening of Transcriptional and Signaling Regulatory Mutant Libraries for Filamentous Growth Defect Under Hypoxia}

To gain insight into regulatory networks that control hyphae formation in response to hypoxia, a compilation of 370 unique mutants of regulatory proteins related to diverse signaling pathways (140 mutants) and transcriptional regulators (230 mutants) from many publicly available libraries were screened (Supplementary Table S1) (Homann et al., 2009; Blankenship et al., 2010; Vandeputte et al., 2012). As sucrose led to enhanced filamentation under hypoxic environments (Mulhern et al., 2006; Askew et al., 2009), our screens were performed using YPS (Yeast-Peptone-Sucrose) medium at $37^{\circ} \mathrm{C}$. Under normoxic conditions, the wild-type $(\mathrm{Wt})$ strain formed colonies with short invasive and aerial filaments, while under hypoxic conditions those filaments are invasive and, at least, four-times longer (Figures 1A, B). The ability of each mutant to form hyphae under hypoxic conditions was assessed by scoring the filamentation of peripheral regions of colonies. Wild type morphology was scored as 0 , reduction of filamentation was scored from -1 to -3 and hyperfilamentation from 1 to 3 . After verifying the observed phenotypes, we have confirmed filamentation defect for 50 mutants in both normoxic and hypoxic conditions. Most of those mutants (38 mutant strains) displayed a reduction of filamentous growth compared to their respective parental strains while 9 strains exhibited enhanced filamentous growth (Figures 1C-E). A total of three mutant strains (ahr1, sch9, tye7) exhibited altered filamentation specifically under a low oxygen environment (Figure 1F).

Mutants of transcriptional regulation showing a filamentous growth alteration both under normoxic and hypoxic conditions included many well-characterized hyphal regulators such as Ndt80, Ssn6, Ace2, Upc2, Cph2, Efg1 and the SAGA complex components Ada 2 and Gcn 5 (Supplementary Table S1). Among mutants with altered hyphal growth, $b c r 1, n d t 80, u p c 2$, age 3 and ace 2 have been already characterized as defectives in filamentation under hypoxic conditions (Mulhern et al., 2006; Lettner et al., 2010; Sellam et al., 2010, 80; Synnott et al., 2011). Filamentation defects of different protein kinase mutants (ire 1, sok1, gin4) reported in the previous large-scale study by Blankenship et al. (Blankenship et al., 2010) under normoxic conditions were also confirmed in our screen. Mutants of other signaling components such as phosphatases (sit4, $p t p 3)$, cell wall sensor $(m s b 2)$, protein acting in the cAMP-mediated (ras 1$)$ and the two-component signaling pathways $(n i k 1, \sin 1)$ were significantly defectives in hyphal growth under both normoxic and hypoxic conditions.

\section{The Transcription Factors Ahr1 and Tye7 Modulate Filamentation Specifically Under Hypoxia}

Among the transcriptional regulators for which we have defined a role in hyphae formation in this study, are the transcription factors Dal81, Mbf1, Yox1 and Fcr3, and the component of the ADA/SAGA complex Ngg1. We also observed hyphal growth 
defects in mutants of diverse signaling pathways, whose role in filamentation was not yet demonstrated (Swe1, Cdc15, Bub1, Ptk2, Kin3, Ckb2, Pho100, Csk1 and Dpl1). Mutants displaying enhanced filamentation specifically under low oxygen environments include $a h r 1$ and tye (Figure 1F). Tye7p and Ahrlp are both transcription factors controlling the expression of glycolytic and adhesin genes, respectively (Askew et al., 2009; Askew et al., 2011). Inactivation of the AGC protein kinase Sch9 led to a hyperfilamentation phenotype comparable to that of ahrl and tye 7 under hypoxia, while under normoxia sch 9 cells were unable to differentiate hyphae (Figure 1F). Earlier works have described the hyperfilamentous phenotype of both tye 7 and sch 9 under hypoxia (Bonhomme et al., 2011; Stichternoth et al., 2011), however, the exact mechanisms by which Tye7 and Sch9 modulate the hypoxic filamentation remain to be determined. For the current study, we decided to focus on Ahrl and Tye7 as they represent potent regulators of hyphal growth specifically under hypoxia. The hyperfilamentous phenotype of both ahr1 and tye 7 was also reproduced by independent mutants made in a different parental background (BWP17) (Supplementary Figure 1). Furthermore, complementation of the $a h r 1$ and tye7 mutants with Wt alleles of AHR1 or TYE7 restored the hypoxic filamentation to a level comparable to that of the Wt BWP17strain.

\section{Transcriptional Program Driving Hypoxic Filamentation}

First, and prior to assessing the contribution of Ahr1 and Tye7 on transcriptional control of the hypoxia-induced filamentation, we wanted to define transcripts that are differentially regulated when $C$. albicans grow as colonies in a solid YPS medium under hypoxic conditions. Differentially expressed genes were

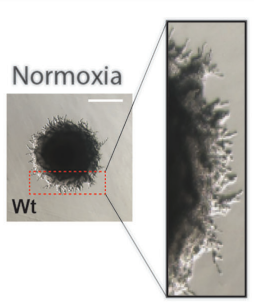

B

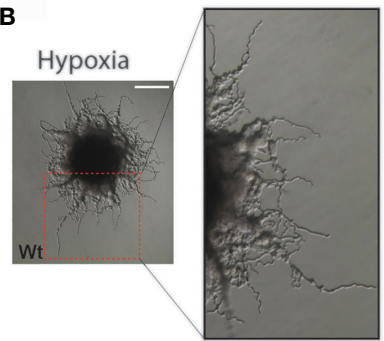

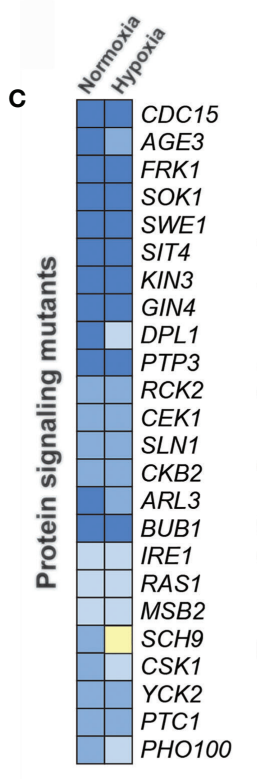
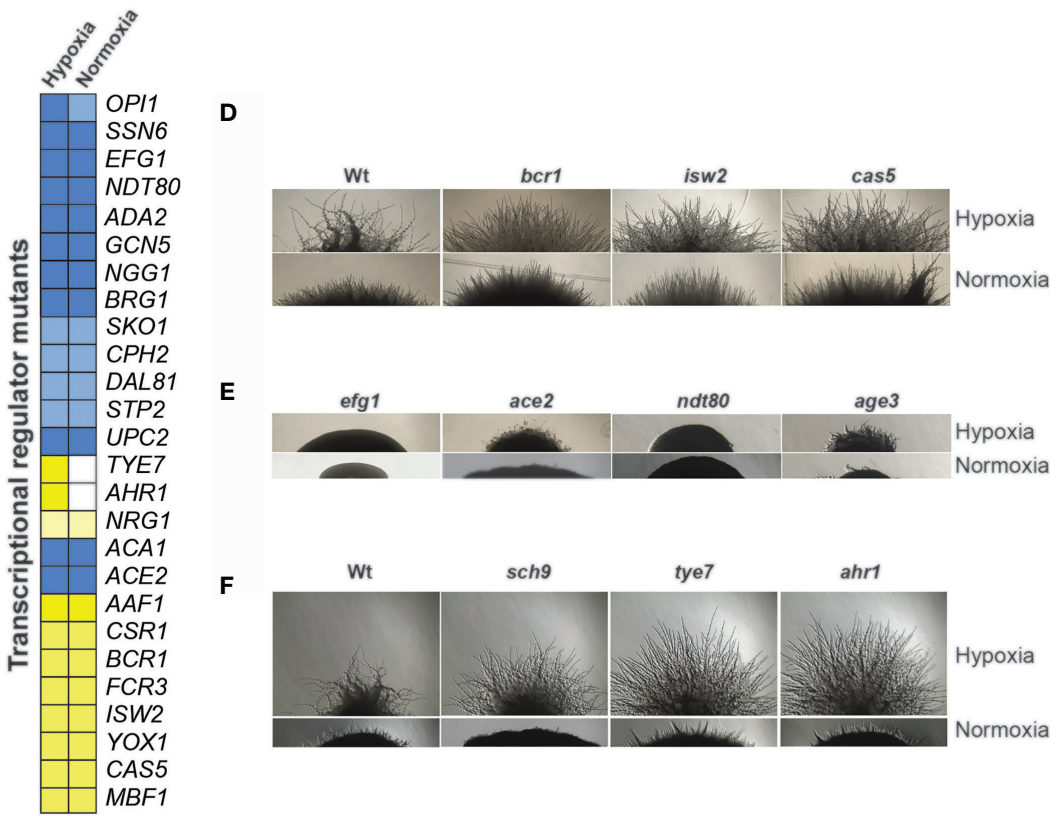

F

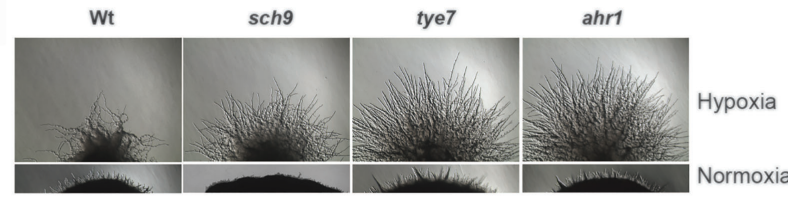

D

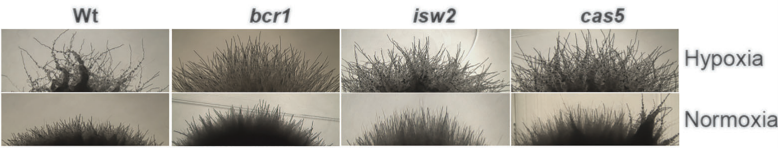

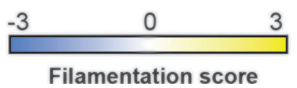

FIGURE 1 | Genetic survey for regulatory proteins required for hypoxic and normoxic filamentation. Morphology of $C$. albicans colony under both normoxia (A) and hypoxia (B) growing in YPS medium at $37^{\circ} \mathrm{C}$. Peripherical regions are magnified and showed short filaments under normoxic conditions (A) while under hypoxia these invasive hyphae are $\sim 4$ times longer (B). (C) Filamentation scoring of the 50 identified mutants. Wt morphology was scored as 0 , reduction of filamentation was scored from -1 to -3 and hyperfilamentation from 1 to 3. (D-F) Single-cell-derived colony morphologies representative of hyperfilamentous (D) and nonfilamentous (F) mutants under either normoxia or hypoxia. (E) Mutants with altered filamentation specifically under hypoxia. Bar, 20 m. 
identified by comparing the transcriptional profiles of colonies exposed to low oxygen concentration $\left(1 \% \mathrm{O}_{2}\right)$ to the transcriptional profiles of colonies growing under normoxic conditions $\left(21 \% \mathrm{O}_{2}\right)$. Using a statistical significance analysis with an estimated false discovery rate of $5 \%$, in addition to a cutoff of 1.5-fold, we identified 365 hypoxic-responsive genes, including 125 upregulated transcripts and 240 downregulated transcripts (Supplementary Table S2). Upregulated genes were significantly enriched in transcripts related to ribosomal biogenesis and rRNA processing as well as metabolic functions such as amino acid and carboxylic acid biosynthesis (Figure 2A). The transcript level of genes related lipid metabolism including ergosterol (ERG1, ERG5, ERG11, ERG13, DAG7, HMG1, IDI1 and CYB5), sphingolipid (SCS7, DES1, SLD1, FEN1 and ARV1), and fatty acids (FAS1, FAS2, FAD2, FAD3 and ALK8) biosynthesis were also significantly induced (Figure $\mathbf{2 A}$ and Supplementary Table S2). Repressed transcripts were enriched in many functions, such as ATP synthesis coupled mitochondrial proton transport (ATP1, ATP2, ATP5, ATP7, ATP14, ATP18 and ATP19) and nucleosome components (HTA2, HHF22, HHF1, HTB1, HTB2 and HTA1) (Figure 2B).

To further mine the transcriptional program underlying hyphae formation under hypoxia, we have used the Gene Set Enrichment Analysis (GSEA) tool (Subramanian et al., 2005; Sellam et al., 2012). GSEA recapitulated the different biological functions altered under the hypoxic filamentation in addition to underlying the carbohydrate genes enrichment among upregulated transcripts (Figure 2C and Supplementary Table S3). The GSEA analysis of the hypoxic filamentation reflected a complex signature that is similar to that experienced by yeast cells growing under similar oxygen status (Setiadi et al., 2006; Synnott et al., 2011; Sellam et al., 2014), and cells undergoing hyphal growth in response to different cues under normoxia (Figures 2D, E). Intriguingly, the enhanced hypoxic filamentation was not accompanied by the activation of the core filamentation genes including the cell wall proteins Hwp1, Als3, Ece1, Ihd1 and Rbt1 (Martin et al., 2013; Azadmanesh et al., 2017). This could be explained by the fact that these transcripts are equally expressed both in our microarrays normoxic control and the hypoxic treatment. A significant similarity was also perceived with cells growing as biofilm confirming the hypoxic environment of this sessile growth of C. albicans (Sellam et al., 2009a) (Figure 2E).

GSEA uncovered that the hypoxic filamentation program was similar to that of mutants of different transcription factors including tye7, upc2 and tbf1 matching their known role in modulating biological processes that were differentially modulated in our experiment including carbohydrate metabolism, ergosterol biosynthesis and translation, respectively (Figure $\mathbf{2 F}$ and Supplementary Table S3). Upregulated transcripts displayed a significant correlation with genes requiring the master filamentation regulator Efg1 for their proper activation. This reflects that the enhanced filamentous growth under hypoxia might be driven by Efg1.

Hypoxic filamentation was accompanied by the activation of genes related to different metabolic processes reflecting a cellular reprogramming of $C$. albicans metabolism to accommodate the metabolic demand of the enhanced hyphal growth (Figure 2 and Supplementary Tables S2, S3). In S. cerevisiae and other yeasts, filamentation allows conquering niches where nutrient conditions are not limiting (Cullen and Sprague, 2012). As hypoxia led to the depletion of many essential metabolites in C. albicans (Burgain et al., 2020), the enhanced filamentous growth might consequently represent a nutritional scavenging response as observed in the budding yeast (Cullen and Sprague, 2012). For instance, oxygen scarcity leads to ergosterol depletion in C. albicans (Burgain et al., 2020) which could serve as a cue to promote hyphal growth. In a support of such hypothesis, genetic perturbation of $E R G$ genes led to a hypofilamentous phenotype comparable to that observed under hypoxia (Uhl et al., 2003).

\section{Transcriptional Profiling of tye7 Mutant Under Hypoxia}

Among the 43-repressed transcripts in tye 7 , a total of 26 were direct targets of Tye7p as previously reported (Askew et al., 2009) (Figure 3A and Supplementary Table S4). This includes glycolytic genes (TPI1, CDC19, GLK1, GPM2, PFK26, PGI1) and genes related to sugar metabolisms (HGT8, OSM1, PDC11, PGM2 and SHA3) (Figures $\mathbf{3 A}, \mathbf{C}$ ). This finding recapitulates the wellknown role of Tye7 as a major transcriptional regulator of carbohydrate metabolism in C. albicans under hypoxia (Askew et al., 2011; Sellam et al., 2014). The hyperfilamentation phenotype of tye 7 might be explained by a reduction of glycolytic flux and impairment of carbon metabolism as previously shown (Askew et al., 2009; Bonhomme et al., 2011).

The 34 transcripts upregulated in tye 7 mutant were enriched in iron utilization genes including the two ferric reductases FRE7 and CFL2, the transcription factor HAP2 and the heme transporter FLC1 (Figure 3A). Tye7 has no apparent role in iron metabolism as it grew normally under iron-depleted media irrespective of oxygen abundance (Supplementary Figure 2). The activation of iron uptake transcripts was previously shown to accompany the yeast-to-hyphae transition and might reflect a phenomenon called adaptive prediction or predictive behaviour (Tagkopoulos et al., 2008; Mitchell et al., 2009; Brunke and Hube, 2014; Roy and Kornitzer, 2019). In C. albicans, such a concept implies a coactivation of process leading to filamentation together with other factors that are required during or after tissue invasion such as iron acquisition. Thus, activation of iron utilization transcripts in tye 7 might reflect such adaptive anticipatory response as this mutant is hyperfilamentous.

\section{Transcriptional Profiling of ahr1 Mutant Under Hypoxia Uncover an Iron Starvation Situation}

Transcripts downregulated in ahrl mutant were enriched mainly in proteolysis (ASI3, DFM1, PRE1, RPN8) (Figures 3B, D and Supplementary Table S4). This pattern might explain the hyperfilamentation phenotype of ahrl since genetic inactivation or pharmacological inhibition of the $C$. albicans proteosome were previously shown to induce filamentation in the absence of an inducing cue (Atir-Lande et al., 2005, 4; Shieh et al., 2005, 4; Kornitzer, 2006; Trunk et al., 2009; Tseng et al., 


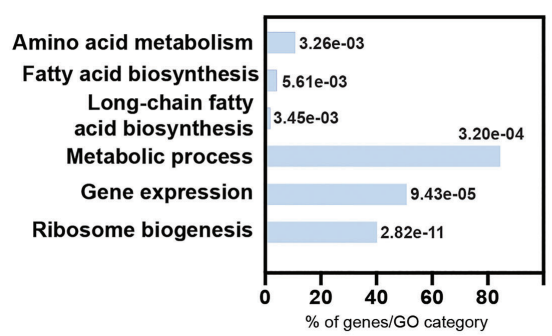

B

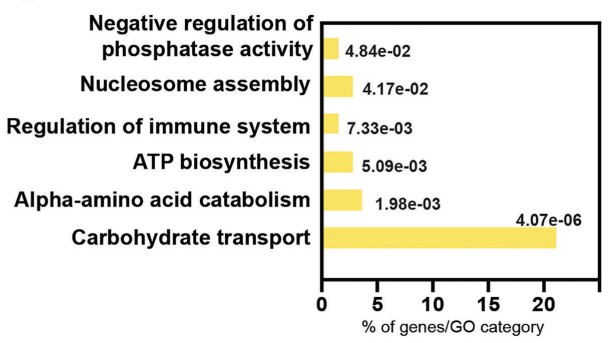

C

Gene ontology_biological process

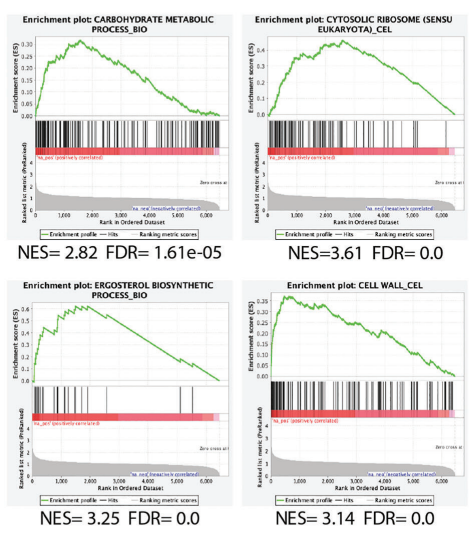

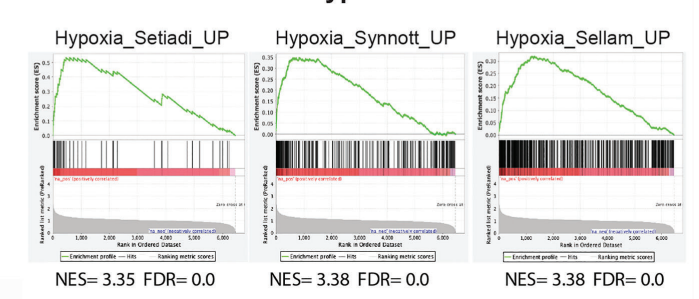

E

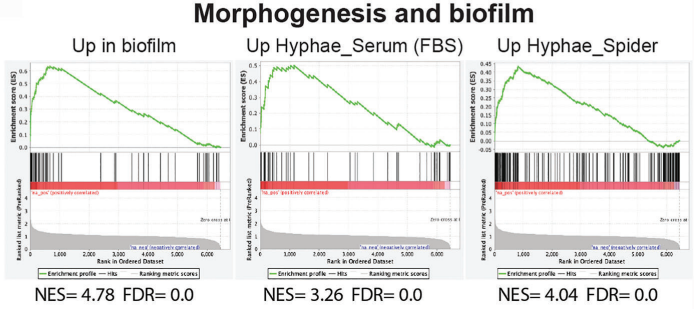

$\mathbf{F}$

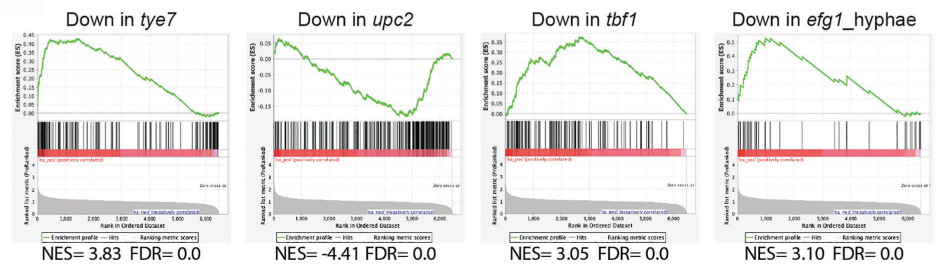

FIGURE 2 | Transcriptional profile driving hypoxic filamentation. Gene ontology analysis of upregulated (A) and downregulated (B) transcripts of C. albicans colonies growing under hypoxia. The $p$-values were calculated using the hypergeometric distribution. (C-F) Gene set enrichment analysis of the transcriptional signatures modulated in response to hypoxia. The complete GSEA correlations are listed in Supplementary Table S3. Graphs of GSEA of relevant correlations with different biological functions (C), former transcriptomics analysis of $C$. albicans growing under hypoxia (D) or under biofilm and hyphal growth states (E), and in different mutant's backgrounds (F). NES, normalized enrichment score; FDR (q-value): False Discovery Rate.

2015; Hossain et al., 2020). Repressed transcripts in ahr1 also included genes related to oxidative stress such as the thioredoxin Trx1, the thiol reductase Ero1 and different proteins with oxidoreductase functions (Cip1, Mxr1, Oye23). Upregulated genes were enriched in function related to mitotic cytokinesis, ATP generation and gene expression regulation (Figure 3D). Activation of cytokinesis transcripts such as those encoding components of the septin ring (Cdc10, Cdc12, Chs1, Int1) and other actomyosin related proteins (Mlc1, Myo2, Dyn1) might mirror the enhanced hyphal growth of ahrl.

Transcript of the multicopper ferroxidase Fet34, a protein that oxidizes $\mathrm{Fe}^{2+}$ to $\mathrm{Fe}^{3+}$ for subsequent cellular uptake by transmembrane permease Ftr1 was highly repressed in ahr1 (Figure 3B). Meanwhile, transcript of Fet31, another multicopper ferroxidase, was induced (Figure 3B) suggesting that Fet31 might be solicited to compensate for the repression of Fet34. While Fet31 was not dispensable for iron uptake in $C$. albicans, Fet34 plays an essential role in iron acquisition (Cheng et al., 2013). Thus, downregulation of Fet34 might reflect an impairment of iron homeostasis in $a h r l$ which could also enable the observed hyperfilementation as previous work showed that iron starvation promotes hyphal growth (Hameed et al., 2008). To test this hypothesis, we first assessed ahrl growth in the presence of the iron-chelating agent BPS. Under yeast-promoting growth 
and either under hypoxia or normoxia, ahrl did not show any perceptible growth defect in the presence of BPS (Supplementary Figure S2). However, ahr1 hyperfilamentation was reverted to a state comparable to that of the Wt strain by supplementing the growth medium with $100 \mu \mathrm{M}$ ferric chloride. This reinforces the hypothesis that the ahrl enhanced hyphal phenotype might reflect a nutritional scavenging response as a consequence of a depleted intracellular iron pool (Figure 3E).

To assess whether the differentially modulated transcripts in ahr1 mutant are direct targets of this transcription factor, Ahr1 occupancy was assessed by ChIP coupled to high-density tiling arrays under hypoxic conditions. Only a few ahrl-misregulated transcripts have their promoter bound by Ahr1 (17/225 differentially expressed genes in $a h r l$ ) (Figure 3B and Supplementary Table S5). This suggests that the observed $a h r 1$ hyperfilamentation phenotype is unlikely the result of expression alteration of Ahr1 direct targets. For instance, neither Fet34 nor proteolysis gene promoters were bound by Ahr1. However, we found that Ahr1 bound the promoter of the heat shock transcription factor Hsf1, a transcriptional modulator of the Hsp90 chaperone network that is a master regulator of morphogenesis in C. albicans (Leach et al., 2016; O'Meara et al., 2017). Former works by the Cowen group uncovered an intriguing phenomenon where either increasing or decreasing HSF1 dosage promote filamentation through two independent mechanisms (Veri et al., 2018). HSF1 overexpression led to transcriptional activation of positive regulators of filamentation, including Brg1 and Ume6 while its depletion compromises Hsp90 function resulting in an increased filamentation. As our microarrays analysis uncovered only a slight induction (1.27-fold change) of HSF1 expression in $a h r 1$ as compared to the $\mathrm{Wt}$, we used qPCR to assess the transcript level of HSF1 in both Wt and ahrl mutant strains. Under normoxia, HSF1 was not modulated in $a h r 1$ as compared to the Wt strain while under hypoxia the transcript level of HSF1 was significantly increased in $a h r 1$ (Figure 3F). This suggest that, under hypoxia, Ahr1 act as a repressor on the promoter of HSF1 which might promote hypoxic filamentation in C. albicans. Thus, Ahr1's role as a repressor of hypoxic filamentation could be mediated through Hsf1 regulatory axis. These observations pave the road for future mechanistic studies investigating the high hierarchical role of Ahr1 as a transcriptional regulator of Hsf1-Hsp90 activity to control hypoxic filamentation. Intriguingly, our omics analysis supports a negative feedback loop control of Ahr1 and Tey7 as they bind the promoter of each other and were required to transcriptionally repress each other (Figures 3A, B).

\section{Genetic Connectivity of the Hypoxic Filamentation Network}

Genetic interactions were used to assess functional relationships between the newly identified modulators of hypoxic filamentation including Ahr1, Tye7 and Sch9. Our data showed that the double mutant ahrltye 7 had an additive phenotype as compared to their congenic strains suggesting that both Ahrl and Tye7 act in parallel pathways (Figure 4A). Deleting SCH9 in either tye 7 or $a h r 1$ resulted in slow-growing smaller colonies which hindered the assessment of filamentation score.
The C. albicans morphogenetic switch is controlled by intertwined regulatory circuits that signal different filamentation cues. We tested the genetic interaction of Ahr1 and Tye7 with the high hierarchical regulators Efgl and the Ras1/Cyr1 cAMP pathway known to promote filamentation in response to a myriad of stimuli (Villa et al., 2020). Genetic inactivation of either Ras1 or Cyr1 in ahrl mutant has no apparent effect while deletion of EFG1 completely abolished the hyperfilamentous phenotype of ahrl (Figure 4B). Contrary, the enhanced hyphal growth of tye 7 mutant under hypoxia was suppressed by either deleting RAS1 or CYR1 but not EFG1 (Figure 4B). Taken together, these data suggest that Ahr1 and Tye7 repress the hypoxic filamentation growth via the Efg1 and Ras1/Cyr1 pathways, respectively. This finding also suggests that both Ahr1 and Tye7 might link the oxygen status of a colonized niche to the general core modulator of $C$. albicans filamentation such as Efg1 and Ras1/Cyr1 cAMP pathways.

In conclusion, the current study uncovered two new regulatory circuits that govern filamentation in response to oxygen levels. Both Tye7 and Ahr1 act as negative regulators of hypoxic filamentation and operate in two independent pathways as supported by our genetic interaction analysis and omics approaches.

\section{MATERIALS AND METHODS}

\section{Strains, Mutant Collections, and Growth Conditions}

The C. albicans strains used in this study were listed in Supplementary Table S6. The kinase (Blankenship et al., 2010) and the transcriptional factor (Homann et al., 2009) mutant collections used for the genetic screens were acquired from the genetic stock center (http://www.fgsc.net). The transcriptional regulator (Vandeputte et al., 2012) mutant collection was kindly provided by Dr. Dominique Sanglard (University of Lausanne). For general propagation and maintenance conditions, the strains were cultured at $30^{\circ} \mathrm{C}$ in a yeast-peptone-dextrose (YPD) medium supplemented with uridine (2\% Bacto-peptone, $1 \%$ yeast extract, $2 \%$ dextrose, and $50 \mu \mathrm{g} / \mathrm{ml}$ uridine). Cell growth and genetic transformation were carried out using standard yeast procedures (Hernday et al., 2010).

For gene expression profiling under hyphae-promoting conditions, cells were collected directly from agar plates with a cell scraper after growing for $48 \mathrm{~h}$ at $37^{\circ} \mathrm{C}$ under either normoxia $\left(21 \% \mathrm{O}_{2}\right)$ or hypoxia $\left(1 \% \mathrm{O}_{2}\right)$. Growth under hypoxic conditions was achieved by incubating agar plates in an anaerobic chamber (Oxoid; HP0011A) continuously flushed with nitrogen to set oxygen levels at $1 \%$ and to remove any gaseous by-products. Collected cells were rapidly frozen in liquid nitrogen and immediately processed for RNA extraction. The effect of iron chelation with BPS (Batho-phenanthroline disulfonic acid) on $C$. albicans growth was performed using spot dilution as described by Khemiri et al. (2020).

\section{Genetic Screen}

For each mutant collection, strains were arrayed using a sterilized 96-well pin tool on Nunc Omni Trays containing YPS-agar and 


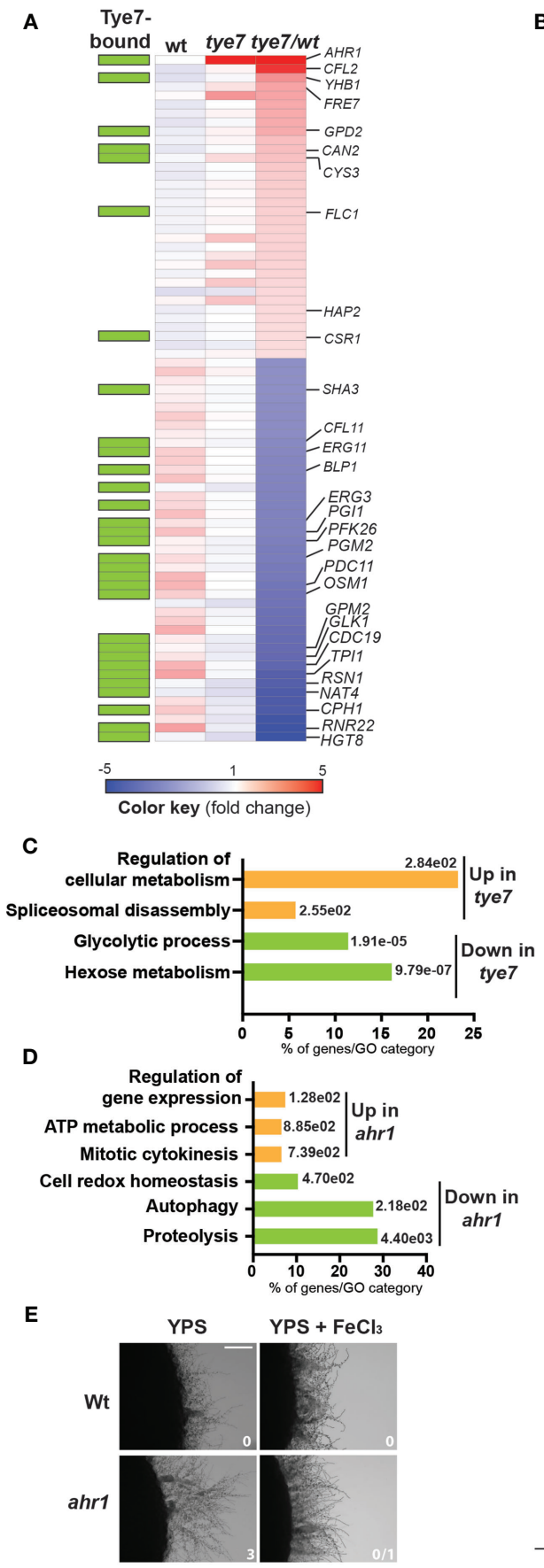

B

Ahr1

bound wt ahr1 ahr1/wt

TYET

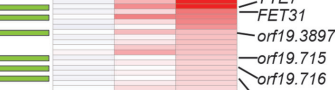

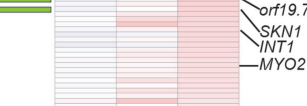

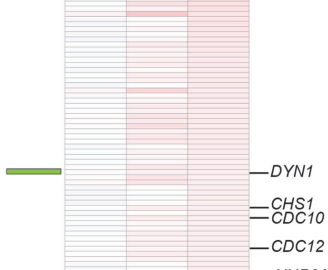

$-N H P C A$
$-M L C 1$
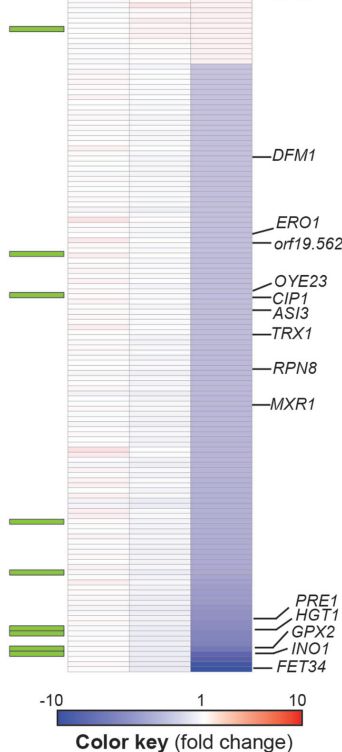

$\mathbf{F}$

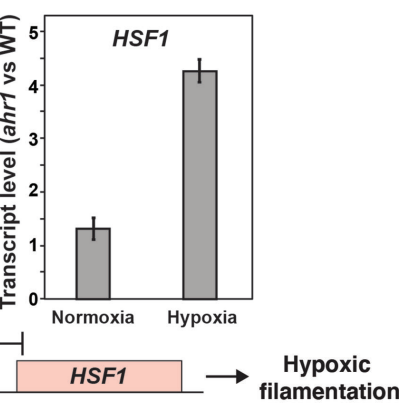

FIGURE 3 | Tye7 and Ahr1 transcription factors act as a negative regulators of $C$. albicans hypoxic filamentation. Heatmap of differentially expressed transcripts in tye7 (A) and ahr1 (B) mutants. Plotted are all transcripts that were significantly differentially expressed (1.5-fold change cut-off and a 5\% FDR) in tye7 and ahr1 mutants. Tye7- and Ahr1-dependant transcripts were identified by comparing the transcriptional profile of tye7 or ahr1 cells to that of Wt cells. Relevant transcripts were annotated in the heatmap. The green boxes indicate gene promoters occupied by Tye7 and Ahr1 as previously defined by Askew et al. (2009) and in the current study, respectively. Blue and red colors represent down- and up-regulated genes, respectively. (C, D) Gene function and biological process enriched in the transcriptional profiles of tye7 (C) and ahr1 (D). The P-value was calculated using a hypergeometric distribution with Multiple Hypothesis Correction, as described on the Gene Ontology (GO) Term Finder website. GO analysis was performed using the Candida Genome Database GO Term Finder. (E) Restauration of the Wt hypoxic filamentation in ahr1 mutant by supplementing YPS-agar medium with $100 \mu \mathrm{M} \mathrm{FeCl}$. Filamentation scores were indicated for each strain and tested condition. Wt and ahr1 strains were grown for 4 days at $30^{\circ} \mathrm{C}$. Bar, $20 \mu \mathrm{m}$. (F) Relative expression of HSF1 in ahr1 mutant. Transcript levels of HSF1 were evaluated in ahr1 cells exposed to normoxia and hypoxia relative to Wt HSF1 levels. Fold-inductions were calculated using the comparative CT method. A model of Ahr1 regulation of hypoxic filamentation through its repressive activity on HSF1 promoter is shown. 
A

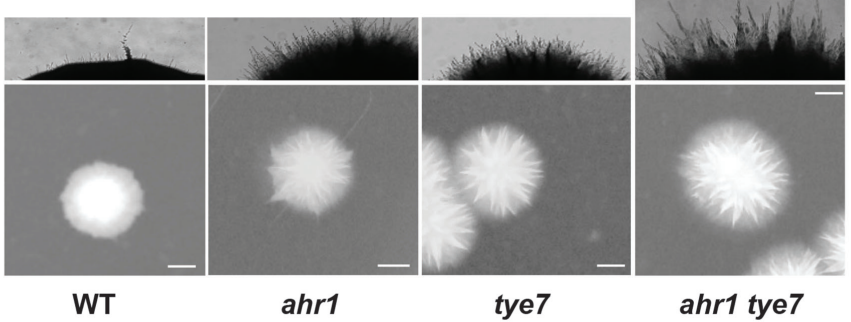

B

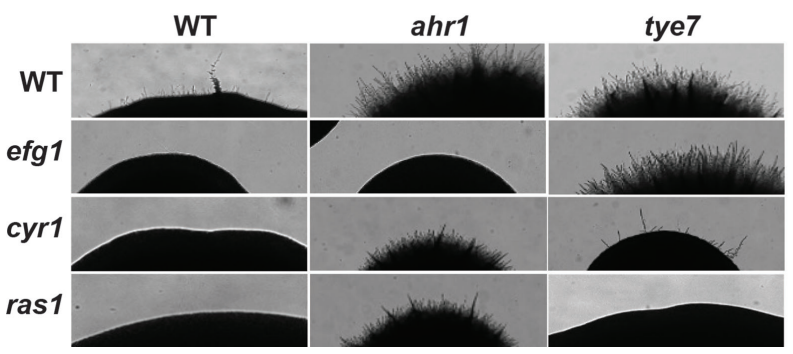

C

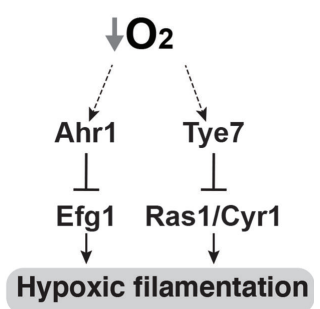

FIGURE 4 | Genetic epistasis of the hypoxic filamentation circuit. (A) Ahr1 and Tye7 act in parallel pathways to modulate hypoxic filamentation. (B) Ahr1 and Tye7 repress the hypoxic filamentous growth via Efg1 and-CAMP-Ras1/Cyr1 pathways, respectively. C. albicans colonies were grown on a YPS-agar medium at $37^{\circ} \mathrm{C}$ under hypoxia $\left(1 \% \mathrm{O}_{2}\right)$ for 3 days. Images of the $\mathrm{Wt}$, and the ahr1 and tye7 mutants in panels (A, B) are the same. (C) Schematic representation of the regulatory network that governs hypoxic filamentation in C. albicans.

colonies were grown for four days at $37^{\circ} \mathrm{C}$ under normoxic $(21 \%$ $\left.\mathrm{O}_{2}\right)$ and hypoxic conditions $\left(1 \% \mathrm{O}_{2}\right)$. Plates were then imaged using the SP-imager system (S\&P Robotics Inc.). A growth score was given for each mutant (Supplementary Table S1). Each mutant hit was confirmed individually by assessing filamentation of at least five single cell-derived colonies.

\section{Expression Analysis by Microarrays and Quantitative-RTPCR}

Total RNA was extracted using an RNAeasy purification kit (Qiagen) and glass bead lysis in a Biospec Mini 24 bead-beater as previously described (Tebbji et al., 2017). RNA was assessed for integrity on an Agilent 2100 Bioanalyzer prior to cDNA labeling. cDNA labeling and microarray procedure were performed as previously described (Sellam et al., 2010, 80). Briefly, $20 \mu \mathrm{g}$ of total RNA was reverse transcribed using $9 \mathrm{ng}$ of oligo $(\mathrm{dT})_{21}$ and $15 \mathrm{ng}$ of random octamers (Invitrogen) in the presence of Cy3 or Cy5-dCTP (Invitrogen) and $400 \mathrm{U}$ of Superscript III reverse transcriptase (ThermoFisher). After cDNA synthesis, template RNA was degraded by adding $2.5 \mathrm{U}$ RNase $\mathrm{H}$ (Promega), and $1 \mu \mathrm{g}$ RNase A (Pharmacia) followed by incubation for $20 \mathrm{~min}$ at $37^{\circ} \mathrm{C}$. The labeled $\mathrm{cDNAs}$ were purified with a QIAquick PCR purification kit (Qiagen). DNA microarrays were processed and analyzed as previously described (Sellam et al., 2009b, 80). To identify transcripts that characterize the hypoxic filamentation in the Wt strain, the transcriptional profile of colonies exposed to low oxygen concentration $\left(1 \% \mathrm{O}_{2}\right)$ was compared to that of colonies growing under normoxic conditions $\left(21 \% \mathrm{O}_{2}\right)$.
Transcript misregulated in tye 7 and ahrl strains were identified by normalizing transcript levels of hypoxiamodulated genes in each mutant against those of the Wt strain (Figures 3A, B and Supplementary Table S4). The GSEA PreRanked tool (http://www.broadinstitute.org/gsea/) was used to determine the statistical significance of correlations between the transcriptome of $C$. albicans hyphal cells under hypoxia with a ranked gene list as previously described (Sellam et al., 2012, 1; Burgain et al., 2019).

For the HSF1 qPCR experiment, a total of two biological and three assay replicates were performed. cDNA was synthesized from $60 \mathrm{ng}$ of total RNA using High-Capacity cDNA Reverse Transcription kit (Applied Biosystems). The mixture was incubated at $25^{\circ} \mathrm{C}$ for $10 \mathrm{~min}, 37^{\circ} \mathrm{C}$ for $120 \mathrm{~min}$ and $85^{\circ} \mathrm{C}$ for $5 \mathrm{~min}$. Two units per microliter of RNAse H (NEB) was added to remove RNA and samples were incubated at $37^{\circ} \mathrm{C}$ for $20 \mathrm{~min}$. qPCR was performed using StepOne ${ }^{\mathrm{TM}}$ Real-Time PCR System (Applied Biosystems) and the PowerUp SYBR Green master mix (Applied Biosystems). The reactions were incubated at $95^{\circ} \mathrm{C}$ for $10 \mathrm{~min}$ and cycled for 40 times at $95^{\circ} \mathrm{C}, 15 \mathrm{~s} ; 60^{\circ} \mathrm{C}, 1 \mathrm{~min}$. Foldenrichments of each tested transcripts were assessed using the $\mathrm{Ct}$ comparative method and actin as a reference gene.

\section{Whole-Genome Location Profiling by ChIP-chip}

Ahr1 was TAP-tagged in vivo with a TAP-HIS1 PCR cassette in SN148 strain as previously described (Lavoie et al., 2008). ChIPchip of Ahr1 under hypoxic conditions was performed using 
tiling arrays as we have previously fulfilled (Sellam et al., 2009b, 80). Briefly, cells were grown as described for the microarrays experiment in YPS-agar plates, harvested with a cell scraper in microcentrifuge tubes and incubated for $20 \mathrm{~min}$ with $1 \%$ formaldehyde for DNA-protein crosslinking. Tiling arrays were co-hybridized with tagged immunoprecipitated (Cyanine 5labeled) and mock immunoprecipitated (untagged SN148 strain; Cyanine 3-labeled) DNA samples. The hybridization was carried out at $42^{\circ} \mathrm{C}$ for $20 \mathrm{~h}$ in a Slide Booster Hybchamber SB800 (Advalytix), with regular micro-agitation of the samples. Slides were washed and air-dried before being scanned using a ScanArray Lite microarray scanner (PerkinElmer). Fluorescence intensities were quantified using ImaGene software (BioDiscovery, Inc.), background corrected and normalized for signal intensity (using Lowess normalization). The significance cut-off was determined using the distribution of log-ratios for each factor which was set at two standard deviations from the mean of log-transformed enrichments. Peak detection was performed using Gaussian edge detection applied to the smoothed probe signal curve, as described by Tuch et al. (Tuch et al., 2008). Both raw and processed microarray and ChIP-chip data have been submitted to the ArrayExpress database at EMBL-EBI (https://www.ebi.ac.uk/arrayexpress/) (Athar et al., 2019) under accession number E-MTAB-10882 and E-MTAB-10883, respectively.

\section{DATA AVAILABILITY STATEMENT}

Strains and plasmids are available upon request. Microarrays and ChIP-chip data have been submitted to the ArrayExpress database at EMBL-EBI (https://www.ebi.ac.uk/arrayexpress/) under accession number E-MTAB-10882 and E-MTAB-10883, respectively.

\section{AUTHOR CONTRIBUTIONS}

Conceptualization: AS. Methodology: MH, AB, FT, and AS. Funding acquisition: AS. Resources: AS. Supervision: AS and FT. Writing original draft: AS. Writing, review, and editing: AS, $\mathrm{MH}, \mathrm{FT}$, and AB. All authors contributed to the article and approved the submitted version.

\section{REFERENCES}

Arkowitz, R. A., and Bassilana, M. (2019). Recent Advances in Understanding Candida Albicans Hyphal Growth. F1000Res 8, F1000 Faculty Rev-700. doi: 10.12688/f1000research.18546.1

Askew, C., Sellam, A., Epp, E., Hogues, H., Mullick, A., Nantel, A., et al. (2009). Transcriptional Regulation of Carbohydrate Metabolism in the Human Pathogen Candida Albicans. PloS Pathog. 5, e1000612. doi: 10.1371/journal.ppat.1000612

Askew, C., Sellam, A., Epp, E., Mallick, J., Hogues, H., Mullick, A., et al. (2011). The Zinc Cluster Transcription Factor Ahrlp Directs Mcmlp Regulation of Candida Albicans Adhesion. Mol. Microbiol. 79, 940-953. doi: 10.1111/j.1365-2958.2010.07504.x

Athar, A., Füllgrabe, A., George, N., Iqbal, H., Huerta, L., Ali, A., et al. (2019). ArrayExpress Update - From Bulk to Single-Cell Expression Data. Nucleic Acids Res. 47, D711-D715. doi: 10.1093/nar/gky964

\section{FUNDING}

This work was supported by funds from the Natural Sciences and Engineering Research Council of Canada discovery fund, the Canadian Foundation for Innovation, the Canadian Institutes for Health Research project grant (CIHR, grant IC118460) and the start-up funds from the Montreal Heart Institute (MHI) to AS. AS is a recipient of the Fonds de Recherche du Québec-Santé (FRQS) J2 salary award.

\section{SUPPLEMENTARY MATERIAL}

The Supplementary Material for this article can be found online at: https://www.frontiersin.org/articles/10.3389/fcimb.2021. 770478/full\#supplementary-material

Supplementary Figure 1 | Confirmation of ahr1 and tye7 hyperfilamentation under hypoxia in the BWP17 strain background. Complementation of the ahr1 (CAS12) and tye7 (CAS3) mutants with Wt alleles of AHR1 or TYE7 restored the hypoxic filamentation to a level comparable to that of the Wt strain (BWP17). Filamentation scores were indicated for each strain. The C. albicans WT, tye7, ahr 1 and the revertant strains were grown on YPS-agar medium and incubated for 6 days at $30^{\circ} \mathrm{C}$ under hypoxic $\left(1 \% \mathrm{O}_{2}\right)$ environments.

Supplementary Figure 2 | The C. albicans Wt, tye7 and ahr1 strains were serially diluted, spotted on YPD-agar medium with different concentrations of BPS and incubated for 2 days at $30^{\circ} \mathrm{C}$ under normoxic $\left(21 \% \mathrm{O}_{2}\right)$ or hypoxic $\left(1 \% \mathrm{O}_{2}\right)$ environments.

Supplementary Table 1 | Raw data of the genetic survey for regulatory proteins required for hypoxic and normoxic filamentation

Supplementary Table 2 | Transcripts differentially expressed in C. albicans Wt (SN250) colonies under hypoxia using a 1.5-fold change cut-off and a 5\% false discovery rate.

Supplementary Table 3 | Gene Set Enrichment Analysis (GSEA) of the transcriptome of C. albicans Wt (SN250) colonies under hypoxia.

Supplementary Table 4 | Transcripts differentially expressed in C. albicans in tye 7 and ahr 1 colonies under hypoxia using a 1.5-fold change cut-off and a 5\% false discovery rate.

Supplementary Table 5 | List of Ahr1-bound intergenic regions as defined by ChIP-tiling arrays.

Supplementary Table 6 | Lists of $C$. albicans strains and primers used in this study.

Atir-Lande, A., Gildor, T., and Kornitzer, D. (2005). Role for the SCFCDC4 Ubiquitin Ligase in Candida Albicans Morphogenesis. MBoC 16, 2772-2785. doi: 10.1091/mbc.e05-01-0079

Austermeier, S., Kasper, L., Westman, J., and Gresnigt, M. S. (2020). I Want to Break Free - Macrophage Strategies to Recognize and Kill Candida Albicans, and Fungal Counter-Strategies to Escape. Curr. Opin. Microbiol. 58, 15-23. doi: 10.1016/j.mib.2020.05.007

Azadmanesh, J., Gowen, A. M., Creger, P. E., Schafer, N. D., and Blankenship, J. R. (2017). Filamentation Involves Two Overlapping, But Distinct, Programs of Filamentation in the Pathogenic Fungus Candida Albicans. G3.: Genes. Genomes. Genet. 7, 3797-3808. doi: 10.1534/g3.117.300224

Bi, S., Lv, Q.-Z., Wang, T.-T., Fuchs, B. B., Hu, D.-D., Anastassopoulou, C. G., et al. (2018). SDH2 Is Involved in Proper Hypha Formation and Virulence in Candida Albicans. Future Microbiol. 13, 1141-1156. doi: 10.2217/fmb-2018-0033 
Blankenship, J. R., Fanning, S., Hamaker, J. J., and Mitchell, A. P. (2010). An Extensive Circuitry for Cell Wall Regulation in Candida Albicans. PloS Pathog. 6, e1000752. doi: 10.1371/journal.ppat.1000752

Bonhomme, J., Chauvel, M., Goyard, S., Roux, P., Rossignol, T., and d'Enfert, C. (2011). Contribution of the Glycolytic Flux and Hypoxia Adaptation to Efficient Biofilm Formation by Candida Albicans. Mol. Microbiol. 80, 9951013. doi: 10.1111/j.1365-2958.2011.07626.x

Brunke, S., and Hube, B. (2014). Adaptive Prediction As a Strategy in Microbial Infections. PloS Pathog. 10, e1004356. doi: 10.1371/journal.ppat.1004356

Brunke, S., Mogavero, S., Kasper, L., and Hube, B. (2016). Virulence Factors in Fungal Pathogens of Man. Curr. Opin. Microbiol. 32, 89-95. doi: 10.1016/ j.mib.2016.05.010

Burgain, A., Pic, É., Markey, L., Tebbji, F., Kumamoto, C. A., and Sellam, A. (2019). A Novel Genetic Circuitry Governing Hypoxic Metabolic Flexibility, Commensalism and Virulence in the Fungal Pathogen Candida Albicans. PloS Pathog. 15, e1007823. doi: 10.1371/journal.ppat.1007823

Burgain, A., Tebbji, F., Khemiri, I., and Sellam, A. (2020). Metabolic Reprogramming in the Opportunistic Yeast Candida Albicans in Response to Hypoxia. mSphere 5, e00913-e00919. doi: 10.1128/mSphere.00913-19

Cheng, X., Xu, N., Yu, Q., Ding, X., Qian, K., Zhao, Q., et al. (2013). Novel Insight Into the Expression and Function of the Multicopper Oxidases in Candida Albicans. Microbiology 159, 1044-1055. doi: 10.1099/mic.0.065268-0

Cullen, P. J., and Sprague, G. F. Jr. (2012). The Regulation of Filamentous Growth in Yeast. Genetics 190, 23-49. doi: 10.1534/genetics.111.127456

Desai, P. R., van Wijlick, L., Kurtz, D., Juchimiuk, M., and Ernst, J. F. (2015). Hypoxia and Temperature Regulated Morphogenesis in Candida Albicans. PloS Genet. 11, e1005447. doi: 10.1371/journal.pgen.1005447

Fisher, M. C., Gurr, S. J., Cuomo, C. A., Blehert, D. S., Jin, H., Stukenbrock, E. H., et al. (2020). Threats Posed by the Fungal Kingdom to Humans, Wildlife, and Agriculture. mBio 11, e00449-e00420. doi: 10.1128/mBio.00449-20

Hameed, S., Prasad, T., Banerjee, D., Chandra, A., Mukhopadhyay, C. K., Goswami, S. K., et al. (2008). Iron Deprivation Induces EFG1 -Mediated Hyphal Development in Candida Albicans Without Affecting Biofilm Formation. FEMS Yeast. Res. 8, 744-755. doi: 10.1111/j.1567-1364. 2008.00394.x

Hernday, A. D., Noble, S. M., Mitrovich, Q. M., and Johnson, A. D. (2010). Genetics and Molecular Biology in Candida Albicans. Methods Enzymol. 470, 737-758. doi: 10.1016/S0076-6879(10)70031-8

Homann, O. R., Dea, J., Noble, S. M., and Johnson, A. D. (2009). A Phenotypic Profile of the Candida Albicans Regulatory Network. PloS Genet. 5, e1000783. doi: 10.1371/journal.pgen.1000783

Hossain, S., Veri, A. O., and Cowen, L. E. (2020). The Proteasome Governs Fungal Morphogenesis via Functional Connections With Hsp90 and cAMP-Protein Kinase A Signaling. mBio 11, e00290-e0220. doi: 10.1128/mBio.00290-20

Jacobsen, I. D., Wilson, D., Wächtler, B., Brunke, S., Naglik, J. R., and Hube, B. (2012). Candida Albicans Dimorphism as a Therapeutic Target. Expert Rev. Anti-infect. Ther. 10, 85-93. doi: 10.1586/eri.11.152

Jr, D. H. B., Giusani, A. D., Chen, X., and Kumamoto, C. A. (1999). Filamentous Growth of Candida Albicans in Response to Physical Environmental Cues and its Regulation by the Unique CZF1 Gene. Mol. Microbiol. 34, 651-662. doi: 10.1046/j.1365-2958.1999.01619.x

Khemiri, I., Tebbji, F., and Sellam, A. (2020). Transcriptome Analysis Uncovers a Link Between Copper Metabolism, and Both Fungal Fitness and Antifungal Sensitivity in the Opportunistic Yeast Candida Albicans. Front. Microbiol. 11, 935. doi: 10.3389/fmicb.2020.00935

Kornitzer, D. (2006). The Ubiquitin System and Morphogenesis of Fungal Pathogens. IMAJ 8, 243-245.

Lavoie, H., Sellam, A., Askew, C., Nantel, A., and Whiteway, M. (2008). A Toolbox for Epitope-Tagging and Genome-Wide Location Analysis in Candida Albicans. BMC Genomics 9:578. doi: 10.1186/1471-2164-9-578

Leach, M. D., Farrer, R. A., Tan, K., Miao, Z., Walker, L. A., Cuomo, C. A., et al. (2016). Hsf1 and Hsp90 Orchestrate Temperature-Dependent Global Transcriptional Remodelling and Chromatin Architecture in Candida Albicans. Nat. Commun. 7, 11704. doi: 10.1038/ncomms11704

Lettner, T., Zeidler, U., Gimona, M., Hauser, M., Breitenbach, M., and Bito, A. (2010). Candida Albicans AGE3, the Ortholog of the S. Cerevisiae ARF-GAPEncoding Gene GCS1, is Required for Hyphal Growth and Drug Resistance. PloS One 5, e11993. doi: 10.1371/journal.pone.0011993
Lu, Y., Su, C., Solis, N. V., Filler, S. G., and Liu, H. (2013). Synergistic Regulation of Hyphal Elongation by Hypoxia, CO2, and Nutrient Conditions Controls the Virulence of Candida Albicans. Cell Host Microbe 14, 499-509. doi: 10.1016/ j.chom.2013.10.008

Martin, R., Albrecht-Eckardt, D., Brunke, S., Hube, B., Hünniger, K., and Kurzai, O. (2013). A Core Filamentation Response Network in Candida Albicans Is Restricted to Eight Genes. PloS One 8, e58613. doi: 10.1371/ journal.pone. 0058613

Mitchell, A., Romano, G. H., Groisman, B., Yona, A., Dekel, E., Kupiec, M., et al. (2009). Adaptive Prediction of Environmental Changes by Microorganisms. Nature 460, 220-224. doi: 10.1038/nature08112

Mulhern, S. M., Logue, M. E., and Butler, G. (2006). Candida Albicans Transcription Factor Ace2 Regulates Metabolism and Is Required for Filamentation in Hypoxic Conditions. Eukaryot. Cell 5, 2001-2013. doi: 10.1128/EC.00155-06

Naglik, J. R., Gaffen, S. L., and Hube, B. (2019). Candidalysin: Discovery and Function in Candida Albicans Infections. Curr. Opin. Microbiol. 52, 100-109. doi: 10.1016/j.mib.2019.06.002

Noble, S. M., French, S., Kohn, L. A., Chen, V., and Johnson, A. D. (2010). Systematic Screens of a Candida Albicans Homozygous Deletion Library Decouple Morphogenetic Switching and Pathogenicity. Nat. Genet. 42, 590598. doi: $10.1038 /$ ng.605

O'Meara, T. R., Robbins, N., and Cowen, L. E. (2017). The Hsp90 Chaperone Network Modulates Candida Virulence Traits. Trends Microbiol. 25, 809-819. doi: 10.1016/j.tim.2017.05.003

Rastogi, S. K., Wijlick, L.v., Ror, S., Lee, K. K., Román, E., Agarwal, P., et al. (2020). Ifu5, a WW Domain-Containing Protein Interacts With Efg1 to Achieve Coordination of Normoxic and Hypoxic Functions to Influence Pathogenicity Traits in Candida Albicans. Cell. Microbiol. 22, e13140. doi: $10.1111 / \mathrm{cmi} .13140$

Roy, U., and Kornitzer, D. (2019). Heme-Iron Acquisition in Fungi. Curr. Opin. Microbiol. 52, 77-83. doi: 10.1016/j.mib.2019.05.006

Sellam, A., Al-Niemi, T., McInnerney, K., Brumfield, S., Nantel, A., and Suci, P. A. (2009a). A Candida Albicans Early Stage Biofilm Detachment Event in Rich Medium. BMC Microbiol. 9, 1-22. doi: 10.1186/1471-2180-9-25

Sellam, A., Askew, C., Epp, E., Tebbji, F., Mullick, A., Whiteway, M., et al. (2010). Role of Transcription Factor CaNdt80p in Cell Separation, Hyphal Growth, and Virulence in Candida Albicans. Eukaryot. Cell 9, 634-644. doi: 10.1128/ EC.00325-09

Sellam, A., Tebbji, F., and Nantel, A. (2009b). Role of Ndt80p in Sterol Metabolism Regulation and Azole Resistance in Candida Albicans. Eukaryot. Cell 8, 11741183. doi: 10.1128/EC.00074-09

Sellam, A., Tebbji, F., Whiteway, M., and Nantel, A. (2012). A Novel Role for the Transcription Factor Cwt1p as a Negative Regulator of Nitrosative Stress in Candida Albicans. PloS One 7, e43956. doi: 10.1371/journal.pone.0043956

Sellam, A., van het Hoog, M., Tebbji, F., Beaurepaire, C., Whiteway, M., and Nantel, A. (2014). Modeling the Transcriptional Regulatory Network That Controls the Early Hypoxic Response in Candida Albicans. Eukaryot. Cell 13, 675-690. doi: 10.1128/EC.00292-13

Sellam, A., and Whiteway, M. (2016). Recent Advances on Candida Albicans Biology and Virulence. F1000Res 5, 2582. doi: 10.12688/f1000research.9617.1

Setiadi, E. R., Doedt, T., Cottier, F., Noffz, C., and Ernst, J. F. (2006), Transcriptional Response of Candida Albicans to Hypoxia: Linkage of Oxygen Sensing and Efglp-Regulatory Networks. J. Mol. Biol. 361, 399-411. doi: 10.1016/j.jmb.2006.06.040

Shapiro, R. S., Ryan, O., Boone, C., and Cowen, L. E. (2012). Regulatory Circuitry Governing Morphogenesis in Saccharomyces Cerevisiae and Candida Albicans. Cell Cycle 11, 4294-4295. doi: 10.4161/cc.22608

Shieh, J.-C., White, A., Cheng, Y.-C., and Rosamond, J. (2005). Identification and Functional Characterization of Candida Albicans CDC4. J. BioMed. Sci. 12, 913-924. doi: 10.1007/s11373-005-9027-9

Spiering, M. J., Moran, G. P., Chauvel, M., Maccallum, D. M., Higgins, J., Hokamp, K., et al. (2010). Comparative Transcript Profiling of Candida Albicans and Candida Dubliniensis Identifies SFL2, a C. Albicans Gene Required for Virulence in a Reconstituted Epithelial Infection Model. Eukaryot. Cell 9, 251-265. doi: 10.1128/EC.00291-09

Stichternoth, C., Fraund, A., Setiadi, E., Giasson, L., Vecchiarelli, A., and Ernst, J. F. (2011). Sch9 Kinase Integrates Hypoxia and CO2 Sensing to Suppress 
Hyphal Morphogenesis in Candida Albicans. Eukaryot. Cell 10, 502-511. doi: 10.1128/EC.00289-10

Subramanian, A., Tamayo, P., Mootha, V. K., Mukherjee, S., Ebert, B. L., Gillette, M. A., et al. (2005). Gene Set Enrichment Analysis: A Knowledge-Based Approach for Interpreting Genome-Wide Expression Profiles. Proc. Natl. Acad. Sci. U.S.A. 102, 15545-15550. doi: 10.1073/pnas.0506580102

Synnott, J. M., Guida, A., Mulhern-Haughey, S., Higgins, D. G., and Butler, G. (2011). Regulation of the Hypoxic Response in Candida Albicans. Eukaryot. Cell 9, 1734-1746. doi: 10.1128/EC.00159-10

Tagkopoulos, I., Liu, Y.-C., and Tavazoie, S. (2008). Predictive Behavior Within Microbial Genetic Networks. Science 320, 1313-1317. doi: 10.1126/science.1154456

Tebbji, F., Chen, Y., Sellam, A., and Whiteway, M. (2017). The Genomic Landscape of the Fungus-Specific SWI/SNF Complex Subunit, Snf6, in Candida Albicans. mSphere 2, e00497-e00417. doi: 10.1128/mSphere.00497-17

Trunk, K., Gendron, P., Nantel, A., Lemieux, S., Roemer, T., and Raymond, M. (2009). Depletion of the Cullin Cdc53p Induces Morphogenetic Changes in Candida Albicans. Eukaryot. Cell 8, 756-767. doi: 10.1128/EC.00332-08

Tseng, T.-L., Lai, W.-C., Lee, T.-L., Hsu, W. H., Sun, Y. W., Li, W.-C., et al. (2015). A Role of Candida Albicans CDC4 in the Negative Regulation of Biofilm Formation. Can. J. Microbiol. 61, 247-255. doi: 10.1139/cjm-2014-0526

Tuch, B. B., Galgoczy, D. J., Hernday, A. D., Li, H., and Johnson, A. D. (2008). The Evolution of Combinatorial Gene Regulation in Fungi. PloS Biol. 6, e38. doi: 10.1371/journal.pbio.0060038

Uhl, M. A., Biery, M., Craig, N., and Johnson, A. D. (2003). HaploinsufficiencyBased Large-Scale Forward Genetic Analysis of Filamentous Growth in the Diploid Human Fungal Pathogen C.Albicans. EMBO J. 22, 2668-2678. doi: $10.1093 / \mathrm{emboj} / \mathrm{cdg} 256$

Vandeputte, P., Pradervand, S., Ischer, F., Coste, A. T., Ferrari, S., Harshman, K., et al. (2012). Identification and Functional Characterization of Rcal, a Transcription Factor Involved in Both Antifungal Susceptibility and Host
Response in Candida Albicans. Eukaryot. Cell 11, 916-931. doi: 10.1128/ EC.00134-12

Veri, A. O., Miao, Z., Shapiro, R. S., Tebbji, F., O’Meara, T. R., Kim, S. H., et al. (2018). Tuning Hsfl Levels Drives Distinct Fungal Morphogenetic Programs With Depletion Impairing Hsp90 Function and Overexpression Expanding the Target Space. PloS Genet. 14, e1007270. doi: 10.1371/journal.pgen.1007270

Villa, S., Hamideh, M., Weinstock, A., Qasim, M. N., Hazbun, T. R., Sellam, A., et al. (2020). Transcriptional Control of Hyphal Morphogenesis in Candida Albicans. FEMS Yeast. Res. 20, foaa005. doi: 10.1093/femsyr/foaa005

Wall, G., Montelongo-Jauregui, D., Vidal Bonifacio, B., Lopez-Ribot, J. L., and Uppuluri, P. (2019). Candida Albicans Biofilm Growth and Dispersal: Contributions to Pathogenesis. Curr. Opin. Microbiol. 52, 1-6. doi: 10.1016/ j.mib.2019.04.001

Conflict of Interest: The authors declare that the research was conducted in the absence of any commercial or financial relationships that could be construed as a potential conflict of interest.

Publisher's Note: All claims expressed in this article are solely those of the authors and do not necessarily represent those of their affiliated organizations, or those of the publisher, the editors and the reviewers. Any product that may be evaluated in this article, or claim that may be made by its manufacturer, is not guaranteed or endorsed by the publisher.

Copyright (c) 2022 Henry, Burgain, Tebbji and Sellam. This is an open-access article distributed under the terms of the Creative Commons Attribution License (CC BY). The use, distribution or reproduction in other forums is permitted, provided the original author(s) and the copyright owner(s) are credited and that the original publication in this journal is cited, in accordance with accepted academic practice. No use, distribution or reproduction is permitted which does not comply with these terms. 